\title{
Hyaluronic Acid-Based Hybrid Hydrogel Microspheres with Enhanced Structural Stability and High Injectability
}

Yun-Jeong Seong, Guang Lin ${ }^{b}$, Byung Jun Kim ${ }^{b}$, Hyoun-Ee Kim ${ }^{a, c}$, Sukwha Kim ${ }^{b, * *}$ Seol-Ha Jeong ${ }^{a, *}$

a Department of Materials Science and Engineering, Seoul National University, Seoul, South Korea

b Department of Reconstructive and Plastic Surgery, Seoul National University Hospital, Seoul, South Korea

c Biomedical Implant Convergence Research Center, Advanced Institutes of Convergence Technology, Suwon, South Korea

* Corresponding authors

** Sukwha Kim: Tel.: +82 22072 3530; fax.: +82 23675 3680;

E-mail address: kimsw@snu.ac.kr

*Seol-Ha Jeong: Tel.: +82 2880 8320; fax.: +82 2884 1413;

E-mail address: jsh528@snu.ac.kr

Keywords: Hybrid hydrogel, Gel microsphere, Injectable filler, Water-in-oil emulsion, Soft tissue engineering 


\section{Supporting Information}

Size distribution of hyaluronic acid hydrogel microspheres and crushed gel particles; Swelling ratio and water contents of bulk gels and crushed gels; Rheological behaviors of the hydrogels; Experimental method and resulting data of immunohistochemical analysis; Optical images of nude mice after hydrogel filler injection

\section{Section $\mathrm{S1}$}
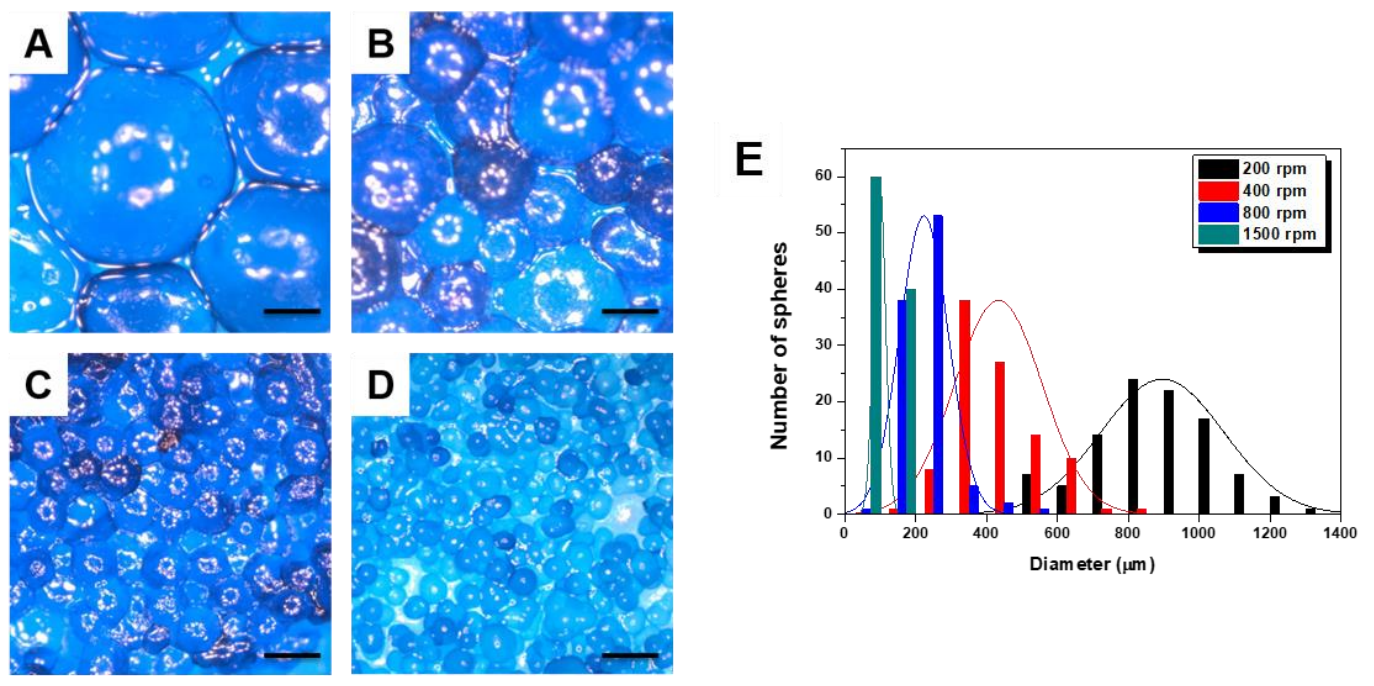

Figure S1. Optic images of hyaluronic acid microspheres fabricated at a rotational speed of (A) $200 \mathrm{rpm}$, (B) $400 \mathrm{rpm}$, (C) $800 \mathrm{rpm}$ and (D) $1500 \mathrm{rpm}$. (E) The size distribution of the microspheres depending on rotational speed. (Scale bars $=\mathbf{4 0 0} \mu \mathrm{m})$

\section{Section S2}



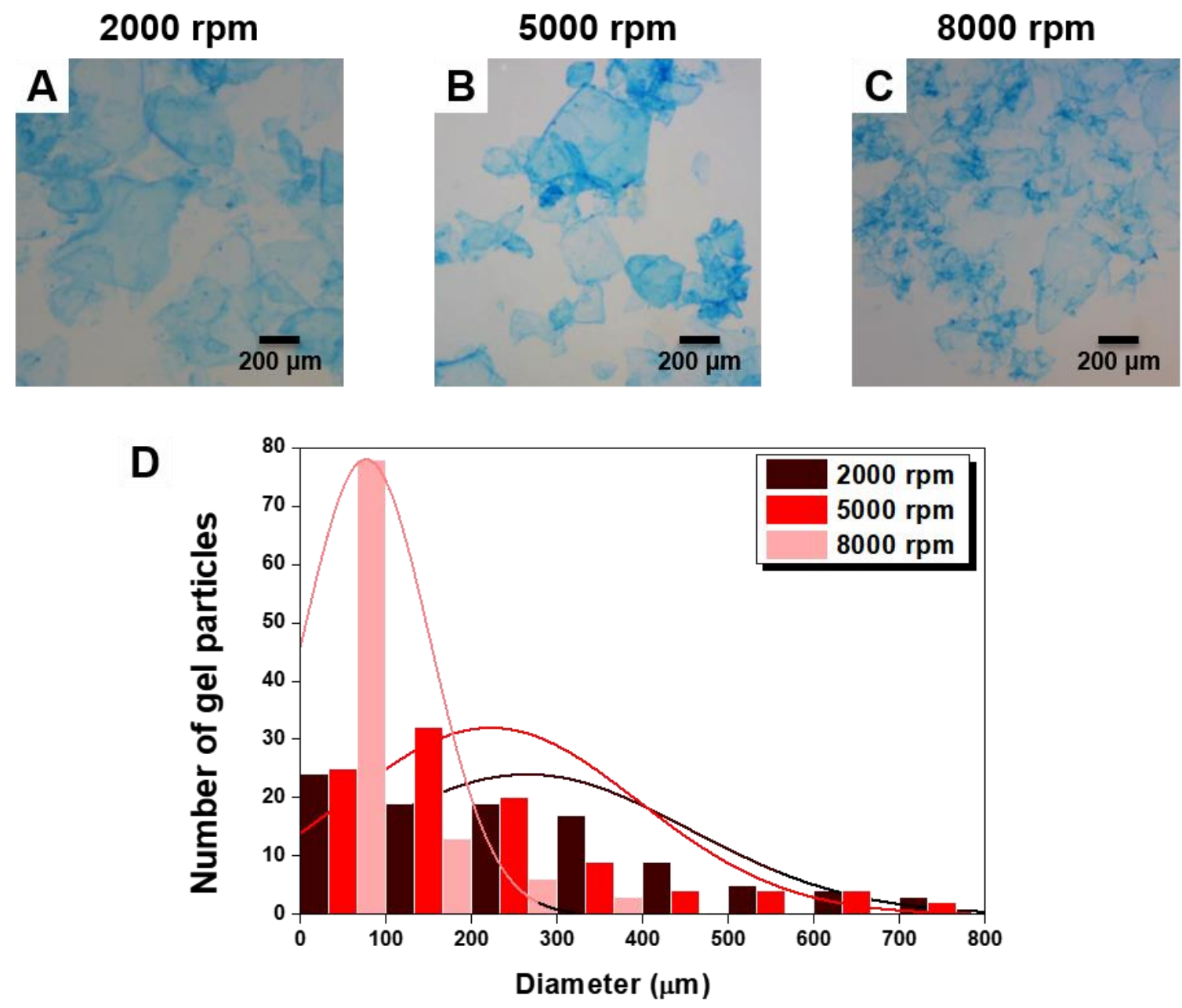

Figure S2. Optical images of crushed hyaluronic acid gel particles prepared with a homogenizer at a rotational speed of (A) $2000 \mathrm{rpm}$, (B) $5000 \mathrm{rpm}$, and (C) $8000 \mathrm{rpm}$. (D) The size distribution of gel particles according to the rotational speed of the homogenizer.

Section S3. 
Table S1. Swelling ratio and water contents of bulk gels and crushed gels

\begin{tabular}{|l|cccc|}
\cline { 2 - 5 } \multicolumn{1}{c|}{} & \multicolumn{2}{c}{ Pure } & \multicolumn{2}{c|}{ Precipitation } \\
\cline { 2 - 5 } & Bulk & Crush & Bulk & Crush \\
\hline Swelling Ratio (g/g) & $36.62 \pm 2.60$ & $35.14 \pm 2.35$ & $24.53 \pm 1.55$ & $25.59 \pm 0.97$ \\
Water content (\%) & $97.33 \pm 0.18$ & $97.23 \pm 0.17$ & $96.07 \pm 0.23$ & $96.24 \pm 0.14$ \\
\hline
\end{tabular}

\section{Section S4}
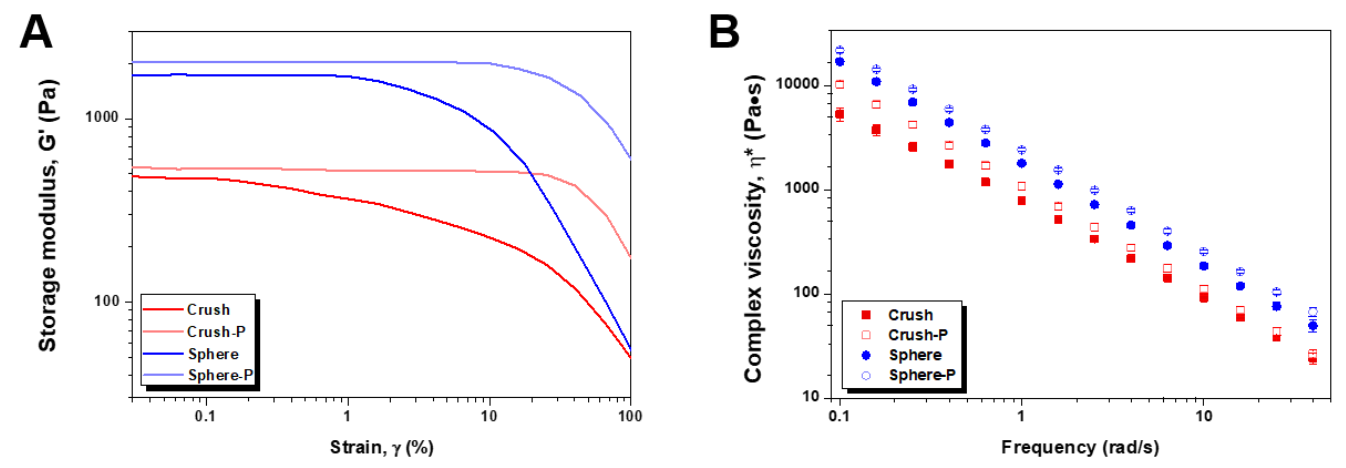

Figure S3. Rheological behavior showing (A) storage modulus for strain sweep and (B) complex viscosity of crushed gels and gel microspheres and each gel with precipitation. The results are presented as the mean \pm SD $(\mathbf{n}=\mathbf{3})$.

\section{Section S5}


The analysis was also carried out on normal nude mice as a control group, in addition to (i) Crush, (ii) Sphere and (iii) Sphere-P. For the experimental groups, the filler injection was performed in 6-week-old mice, and sacrifice and tissue analysis were performed after 12-week follow-up. Therefore, for the control group, 18-week old mice were sacrificed and the same analysis was performed.

The experiment was carried out as follows;

The expression of CD68 was also analyzed using immunohistochemical staining to verify the degree of immune response. Briefly, the paraffin sections were deparaffinized and rehydrated followed by antigen retrieval in $10 \mathrm{mM}$ sodium citrate buffer for $30 \mathrm{~min}$ at $90{ }^{\circ} \mathrm{C}$ and treatment with $0.3 \mathrm{v} / \mathrm{v} \%$ hydrogen peroxide. After blocking in $10 \mathrm{v} / \mathrm{v} \%$ normal serum with $1 \mathrm{w} / \mathrm{v} \% \mathrm{BSA}$ in tris-buffered saline for $2 \mathrm{~h}$ at room temperature, the sections were incubated with anti-CD68 antibody overnight at $4{ }^{\circ} \mathrm{C}$. Next, the sections were washed with $1 \mathrm{v} / \mathrm{v} \%$ Triton $\mathrm{X}-100$ in PBS and incubated with goat anti-rabbit secondary antibody for $2 \mathrm{~h}$ at room temperature. Then, the slides were treated with 3,3'-diaminobenzidine and dehydrated before being observed and analyzed using the Image $\mathrm{J}$ program. 


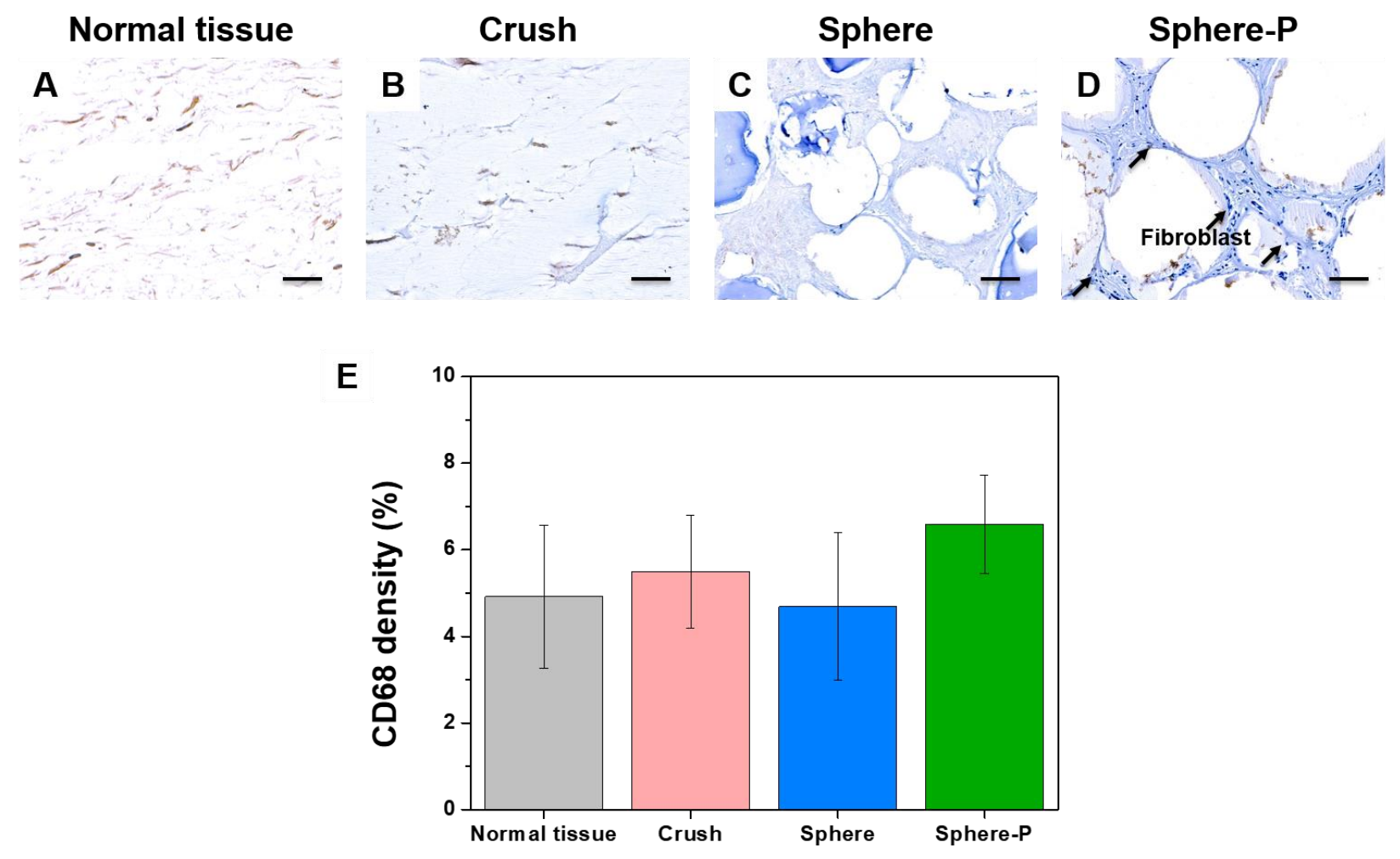

Figure S4. Immunohistochemical analysis using anti-CD68 antibody after 12 weeks of injection. Immunhistochemically stained images of (A) normal tissue and tissue injected with (B) crushed gels, (C) gel microspheres, and (D) gel microspheres with precipitation. (E) CD68 expression in ingrowth tissue. The results are presented as the mean \pm SD $(n=3) .($ Scale bars $=50 \mu \mathrm{m})$ 


\section{Section S6}

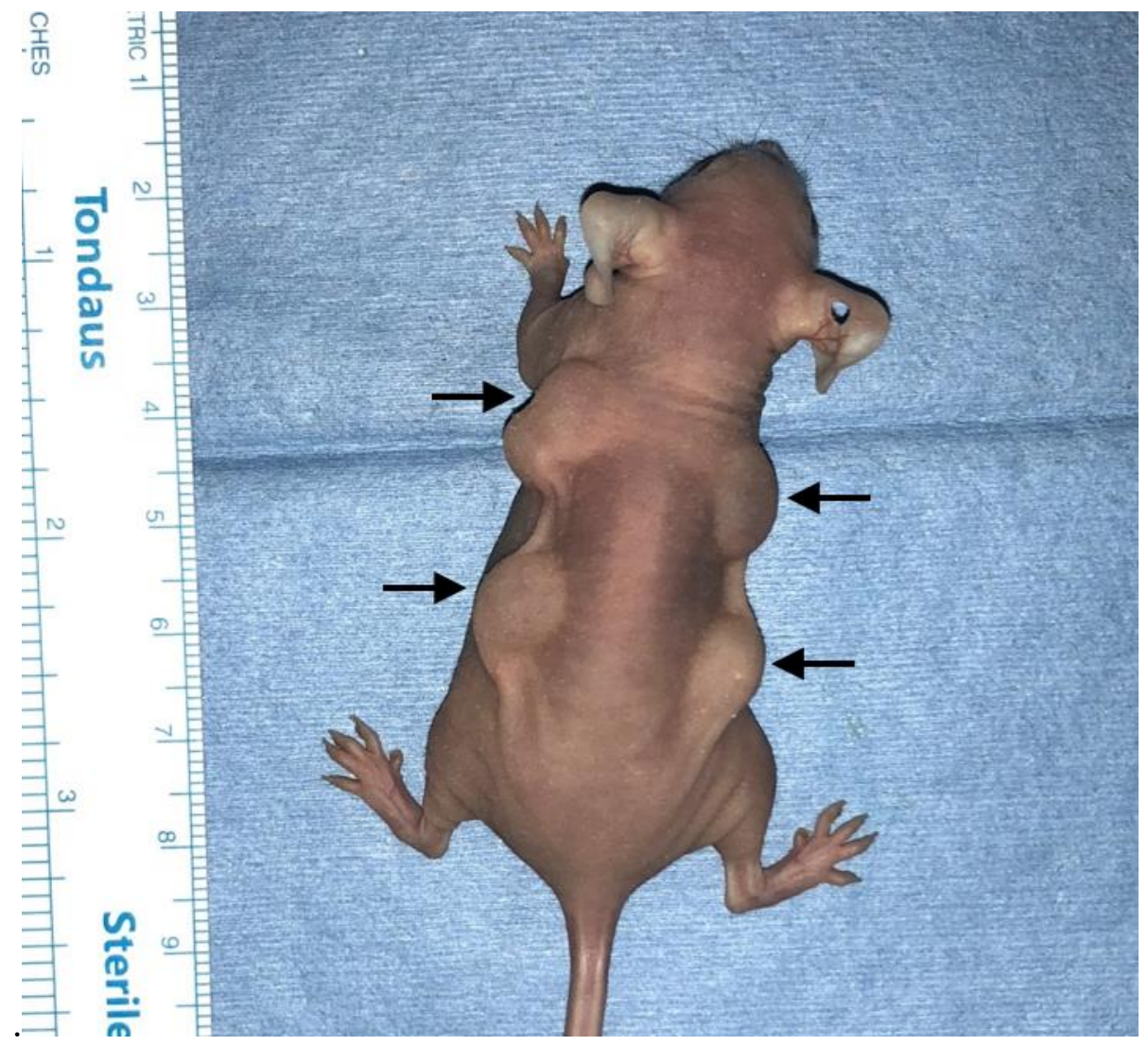

Figure S5. Macroscopic appearance of the nude mice immediately after injecting $200 \mu \mathrm{l}$ of gel into four places of the back. (Arrows: injected gel) 